\title{
Identification of fimbrial subunits in the genome of Trueperella pyogenes and association between serum antibodies against fimbrial proteins and uterine conditions in dairy cows
}

\author{
R. S. Bisinotto, ${ }^{*}$ J. C. Oliveira Filho, ${ }^{*}$ C. Narbus, ${ }^{*}$ V. S. Machado, ${ }^{*}$ E. Murray, $\dagger$ and R. C. Bicalho ${ }^{* 1}$ \\ ${ }^{*}$ Department of Population Medicine and Diagnostic Sciences, College of Veterinary Medicine, and \\ †Department of Entomology, College of Agriculture and Life Sciences, Cornell University, Ithaca, NY 14853
}

\begin{abstract}
Understanding the role of fimbrial subunits during bacterial adherence and the host's immunological response against anchorage proteins is critical for the development of strategies to prevent pathogens from thriving. The objectives of the present study were to locate fimbria-related proteins in the genome of Trueperella pyogenes (CP007519), define their importance for bacterial adherence, and evaluate the association between serum antibodies against fimbrial subunits and uterine health in dairy cows. Using a BLASTp search through the GenBank database, 4 putative clusters for fimbrial assembly were identified in the genome of $T$. pyogenes, namely FimA, FimC, FimE, and the novel major fimbriae FimJ. The fimbrial proteins FimA, FimC, FimE, and surface-anchored protein (SAP) were cloned into the $\mathrm{pET} 26 \mathrm{~b}(+)$ vector, expressed in Escherichia coli BL21, and purified using affinity chromatography. Serum antibodies against FimA, FimC, FimE, and SAP were determined by ELISA on d $260 \pm 3$ of gestation and at $2 \pm 1$ and $35 \pm$ $3 \mathrm{~d}$ in milk (DIM) to assess the relationship between antigenicity against fimbrial proteins and parameters of uterine health. Antibodies against FimC and FimE were greater both pre- and postpartum in cows from which $T$. pyogenes was recovered by uterine flushing at $35 \pm 3$ DIM, whereas $T$. pyogenes infection was not associated with differences in serum concentrations of FimA and SAP antibodies. Likewise, concentrations of FimC antibodies were consistently greater in cows diagnosed with clinical endometritis at $35 \pm 3$ DIM compared with healthy counterparts. These results suggest that fimbrial proteins evaluated in the present study, particularly FimC and FimE, are important for maintenance of $T$. pyogenes in the uterus postpartum and development of uterine diseases in dairy cattle. Ad-
\end{abstract}

Received September 15, 2015.

Accepted January 16, 2016.

${ }^{1}$ Corresponding author: rcb28@cornell.edu ditional research is warranted to elucidate the mechanisms by which each fimbrial subunit contributes to the establishment of uterine diseases, evaluate its effect on fertility responses, and assess its relevance as a target for vaccine development.

Key words: metritis, endometritis, fimbria, immune response

\section{INTRODUCTION}

Trueperella pyogenes is a gram-positive bacteria commonly isolated from the udder, upper respiratory, urogenital, and gastrointestinal tracts of livestock (Jost and Billington, 2005). Despite being a commensal microorganism in cattle, T. pyogenes is an opportunistic pathogen and has been implicated in a multitude of health disorders, such as mastitis, uterine diseases, pneumonia, and liver abscedation (Doré et al., 2007; Machado et al., 2012a; Ribeiro et al., 2015). Extensive research has been conducted to evaluate the role of T. pyogenes during establishment of uterine diseases in dairy cows, mostly due to negative effects on reproductive efficiency (Williams et al., 2005). The prevalence of T. pyogenes in cows diagnosed with metritis during the first $2 \mathrm{wk}$ of lactation is similar to that observed in healthy herdmates (Bicalho et al., 2012; Machado et al., 2012a). Nevertheless, T. pyogenes persistency leads to subclinical and clinical endometritis (Machado et al., 2012a; Sens and Heuwieser, 2013) and prolonged time to pregnancy (Williams et al., 2005).

Although the tissue damage associated with T. pyogenes infection is caused by a cholesterol-dependent cytolysin named pyolysin (Jost et al., 1999; Amos et al., 2014), it is likely that anchorage proteins also play a major pathogenic role. For instance, adhesion to host cells is required for colonization of the mucosa and progression of diseases caused by other gram-positive bacteria (Mandlik et al., 2007; Mandlik et al., 2008). Several mechanisms are involved with adhesion of $T$. pyogenes to host cells including neuraminidases (Jost and Billington, 2005), collagen-binding proteins (Esmay 
et al., 2003), and fimbriae (Machado and Bicalho, 2014; Goldstone et al., 2014). Previous studies have depicted the importance of a fimbrial gene, namely fimA, for the development of metritis and endometritis in dairy cows (Santos et al., 2010; Bicalho et al., 2012). Because cell surface proteins mediate the interaction between host and pathogens, they provide a key signature for elimination of infection by immune cells. Accordingly, the identification and characterization of antigenic epitopes is critical for the development of vaccines against gram-positive bacteria (Maione et al., 2005; Nuccitelli et al., 2013). Poor fimbriae expression in vitro by wild strains of $T$. pyogenes is a major impediment for the characterization of its adhesion mechanisms. However, the publication of the $T$. pyogenes complete genome (Goldstone et al., 2014; Machado and Bicalho, 2014) offers a unique platform for localization and in-depth analyses of fimbrial genes. Identification of epitopes harbored in the fimbrial structures of $T$. pyogenes is expected to provide novel targets for vaccine development against uterine diseases (Machado et al., 2014b).

Therefore, the overall objective of the present study was to explore the relevance of $T$. pyogenes fimbriae in dairy cows. The study encompasses 3 specific objectives, namely to (1) examine the genome of $T$. pyogenes TP6357 (GenBank accession number CP007519) for the presence of major fimbrial subunit genes, (2) build a recombinant model for expression of $T$. pyogenes fimbrial subunits in vitro, and (3) evaluate temporal changes in antigenicity against $T$. pyogenes fimbrial subunits in dairy cows with different uterine conditions postpartum. We hypothesized that antigenicity against fimbrial proteins encoded in the genome of $T$. pyogenes is associated with the presence of pathogenic bacteria in the uterus and incidence of uterine diseases in lactating dairy cows.

\section{MATERIALS AND METHODS}

Three complementary studies were conducted and each one is presented under a different subheading in the Materials and Methods section. In the first study, genomic methods were applied to identify clusters of fimbrial assembly in the genome of T. pyogenes TP6357, which has been isolated from the uterus of a dairy cows diagnosed with metritis. In the second study, an in vitro model for expression and purification of recombinant fimbrial proteins was designed. Finally, the presence of serum antibodies against fimbrial subunits was evaluated in the third study as an assessment of their involvement during the development of different uterine conditions in dairy cows postpartum.

\section{Characterization of Major Fimbrial Subunit Genes in the Genome of T. pyogenes}

Gene Localization. The complete genome sequence of T. pyogenes strain TP6357 has been deposited in GenBank under the accession number CP007519 (Machado and Bicalho, 2014). This strain was used in the present study because it was isolated from the uterus of a dairy cow diagnosed with metritis, defined based on the presence of fetid, watery, red-brown vaginal discharge retrieved after transrectal massage of the uterus associated with rectal temperature greater than $39.5^{\circ} \mathrm{C}$. Open reading frame ( ORF) numbers for $T$. pyogenes TP6357 have been generated previously using the NCBI Prokaryotic Annotation Pipeline (Machado and Bicalho, 2014). Initial screening for fimbrial subunits was performed using Geneious version 8.0.4 (Biomatters, Auckland, New Zealand) according to primer sequences published elsewhere (Silva et al., 2008). Additionally, putative genes encoding for major fimbriae were selected based on the presence of cell-wall sorting signals (CWSS), fimbrial motifs, and E box sequences, all of which are conserved sequences associated with fimbrial clusters. Coding sequences defined using this pipeline and adjacent coding sequences were translated using the protein basic local alignment search tool (BLASTp) against the nonredundant GenBank database (www.ncbi.nlm.nih.gov/genbank) for prediction of putative functions and identification of putative fimbrial operons.

Sequence Alignment and Phylogenetic Analysis. Because putative major fimbrial genes are hypothesized paralogs, the evolutionary relationships among these regions were reconstructed to assess their connection both in T. pyogenes and related taxa. A BLASTp search was performed in the GenBank database to identify sequences closely related to fimbrial gene regions from T. pyogenes TP6375. The top 9 matches for each region were selected based on their E-value and downloaded for subsequent analyses. Gene regions were aligned using the MAFFT v7 online server with the E-INS-i strategy (Katoh and Standley, 2013). Duplicate species for each Fim region were removed. For maximum likelihood analyses, the CIPRES v3.3 interface (Miller et al., 2015) was used to access RAxML v8.1.11 (Stamatakis, 2014), employing the BLOSUM substitution matrix. Branch support was assessed using 1,000 rapid bootstrap replicates. Gblocks $0.91 \mathrm{~b}$ was also used for algorithmic alignment masking (Talavera and Castresana, 2007). Alignment masking was used to increase the signal-to-noise ratio by removing regions of ambiguous and uncertain alignment, as described by Kück et al. (2010). 


\section{Production of T. pyogenes Recombinant Fimbrial Proteins}

Plasmid Construction. Trueperella pyogenes fimA, fim $C$, fimE, and the sequence encoding for surfaceanchored protein (SAP) located close to fim $C$ were cloned into the $\mathrm{pET} 26 \mathrm{~b}(+)$ vector (Novagen, Madison,

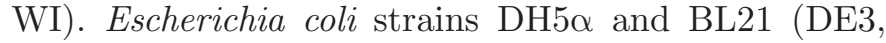
Agilent Technologies, Santa Clara, CA) were used for plasmid extraction and protein expression, respectively. All proteins were cloned without the signal peptide, which was identified using SignalP 4.1 server (Petersen et al., 2011). Plasmids were constructed by PCR amplification of the target genes from genomic DNA using the primers listed in Table 1. Each PCR product was TA cloned into a pGEM T vector (Promega, Madison, WI) and blue/white screening was used to select positive clones. Plasmids from positive clones were digested with the respective restriction enzymes and ligated into a digested pTrcHis A vector (Invitrogen, Carlsbad, CA). All constructs were confirmed by Sanger sequencing.

Protein Expression and Purification. Single colonies were inoculated into Luria-Bertani broth containing $30 \mu \mathrm{g} / \mathrm{mL}$ of kanamycin and incubated at $37^{\circ} \mathrm{C}$ under constant shaking at $200 \mathrm{rpm}$ overnight. Following the incubation period, $0.4 \mathrm{~mL}$ of growth culture was transferred to $40 \mathrm{~mL}$ of fresh medium under the same conditions and $1 \mathrm{~m} M$ of isopropyl $\beta$-D-1-thiogalactopyranoside (IPTG; IBI Scientific, Peosta, IA) was added when the culture reached an optical density at $600 \mathrm{~nm}\left(\mathrm{OD}_{600}\right)$ of 0.6 for induction of protein expression. Cells were harvested at $4 \mathrm{~h}$ after induction. Samples were centrifuged at $10,000 \times g$ for 5 min at room temperature. Cell pellets were resuspended in lysis buffer (10 mM Tris-HCl; $1 \mathrm{~m} M$ EDTA; $0.1 \mathrm{~N}$ $\mathrm{NaOH} ; 0.5 \% \mathrm{SDS}$ ) and centrifuged at $13,000 \times g$ and $4^{\circ} \mathrm{C}$ for $10 \mathrm{~min}$ for removal of insoluble proteins and cell debris. The supernatant was boiled with Laemmli buffer (63 m $M$ Tris-HCl pH 6.8, 10\% glycerol, $2 \%$ SDS, $0.1 \% \beta$-mercaptoethanol, $0.0005 \%$ bromophenol blue) for 5 min and loaded on to a $12 \%$ SDS-polyacrylamide gel. The gel was run at $80 \mathrm{~V}$ for 90 min using the MiniProtean Tetra Cell electrophoresis system (Bio-Rad, Hercules, CA) and stained (Coomassie Brilliant Blue R-250 Staining Solution, Bio-Rad) for 40 min. After staining was completed, destaining was performed for $3 \mathrm{~h}$ under vigorous shaking (Coomassie Brilliant Blue R-250 Destaining Solution, Bio-Rad). The gel was then visualized using the MiniBIS Pro (DNR Bio-Imaging Systems, Jerusalem, Israel) with Gel capture software to digitalize the gel image. Recombinant proteins containing a 6 -His tag on carboxyl termini were purified using TALON metal affinity resin (Clontech Laboratories Inc., Mountain View, CA). For production of recombinant proteins, cells were cultured as described above. After overnight incubation, bacteria were harvested by centrifuging $8 \mathrm{~L}$ of culture medium at $10,000 \times g$ for 10 min at $4^{\circ} \mathrm{C}$. Cells were resuspended by adding $1 \%$ of the original volume of buffer A (50 $\mathrm{m} M$ of sodium sulfite phosphate $\mathrm{pH} 7.0,300 \mathrm{~m} M$ of $\mathrm{NaCl}$ ). Cell suspension was incubated on ice for $30 \mathrm{~min}$ with $0.75 \mathrm{mg} / \mathrm{mL}$ of lysozyme (Sigma-Aldrich, St. Louis, MO). A protease inhibitor cocktail $(0.1 \% \mathrm{vol} / \mathrm{vol}$; Invitrogen $)$ was added to the medium and cells were lysed by 2 passages through a French Press cell (Aminco, Lake Forest, CA) at 20,000 psi (138 MPa). Lysed cells were centrifuged at $12,000 \times g$ and $4^{\circ} \mathrm{C}$ for $45 \mathrm{~min}$ and the supernatant was poured into centrifuge bottles containing $1 \mathrm{~mL}$ of TALON resin per liter of culture pre-equilibrated with buffer A. The bottles were incubated overnight on a rotating table at $4^{\circ} \mathrm{C}$. The mixture was washed twice using $20 \mathrm{~mL}$ of buffer A per $\mathrm{mL}$ of resin and beds were resuspended in $2 \mathrm{~mL}$ of buffer $\mathrm{A}$ per $\mathrm{mL}$ of resin to pack a column by gravity. Resin centrifugation steps were completed at $1,000 \times g$ and $4^{\circ} \mathrm{C}$ for $5 \mathrm{~min}$. Elution was performed with $10 \mathrm{~mL}$ of buffer B $(50 \mathrm{~m} M$ sodium sulfite phosphate $\mathrm{pH} 7.0,300 \mathrm{~m} M \mathrm{NaCl}, 150$

Table 1. Primers used for plasmid construction ${ }^{1}$

\begin{tabular}{lll}
\hline Gene & Primer sequence & $\begin{array}{l}\text { Restriction } \\
\text { enzyme }\end{array}$ \\
\hline fimA & F: 5'-CCTCGAGAAGCCGAGCGGATTTCCTGTTG & XhoI \\
& R: 5'-GAAGCTTTTAGGCATTACGACGACGAGCGAC & HindIII \\
fim C & F: 5'-CGGATCCGATACACAGTCTGGTTTCAATGC & BamHI \\
& R: 5'-GAAGCTTTTAGCTACGACGACGAGCGTG & HindIII \\
fimE & F: 5'-CGGATCCGCGCCAGCGCCCAAACCGCAGG & BamHI \\
SAP & R: 5'-GAAGCTTTTAGACGTCCTTGCGTCGGTT & HindIII \\
& F: 5'-CGAGCTCACAGCCGCGTTATGCATATCG & SacI \\
& R: 5'-CTCGAGCTACGATCCGAGTCGCCGCTTGC & XhoI \\
\hline${ }^{1}$ All primers were designed for this study. Restriction enzyme recognition sites are underlined in each sequence.
\end{tabular}

$\mathrm{F}=$ forward; $\mathrm{R}=$ reverse. 
$\mathrm{m} M$ imidazole) per $\mathrm{mL}$ of resin. Purified proteins were concentrated using centrifugal filters with a $10-\mathrm{kDa}$ cutoff membrane (EMD Millipore, Billerica, MA). Final protein concentration was determined by Bradford assay (Protein Assay Dye Reagent Concentrate, BioRad) and albumin was used as reference. Recombinant proteins were stored at $-20^{\circ} \mathrm{C}$ with $50 \%$ of glycerol. Protein yield was $5.1,3.7,4.7$, and $4.1 \mathrm{mg} / \mathrm{L}$ of culture for FimA, FimC, FimE, and SAP, respectively. Molecular weight observed in the SDS-gel were approximately 55, 55, 64, and $95 \mathrm{kDa}$ for FimA, FimC, FimE, and SAP, respectively, which was expected based on the number of AA residues for each protein.

\section{Assessment of Antigenicity to T. pyogenes Fimbrial Proteins in Dairy Cows}

Analyses of Serum Antibodies Against Fimbrial Subunits by ELISA. Sera from 81 cows were assessed for the presence of natural antibodies against T. pyogenes fimbriae by ELISA. Blood from nulliparous Holstein heifers was sampled at $260 \pm 3 \mathrm{~d}$ of gestation and again at $2 \pm 1$ and $35 \pm 3$ DIM (i.e., primiparous cows), and henceforth is referred to as $\mathrm{d}-30,2$, and 35 relative to calving. Blood was collected by puncture of coccygeal vessels into evacuated tubes (Vacutainer; Becton Dickinson, Franklin Lakes, NJ). Serum was harvested by centrifugation at 2,000 $\times \mathrm{g}$ for $15 \mathrm{~min}$ at $4^{\circ} \mathrm{C}$ and stored at $-80^{\circ} \mathrm{C}$ until assayed. Recombinant fimbrial proteins FimA, FimC, FimE, and SAP were used as antigens for measurement of serum IgG by ELISA. Microtiter plates (Greiner Bio-One, Monroe, NC) were coated with $4 \mu \mathrm{g}$ of FimA, FimC, FimE, or SAP diluted in $100 \mu \mathrm{L}$ of sterile PBS (Ambion Life Technologies, Grand Island, NY). Binding of antigen to microplate wells were carried out overnight at $4^{\circ} \mathrm{C}$. Serum samples were diluted to 1:200 for the assay. Target antibodies bound to the ELISA plate were detected with anti-bovine IgG conjugated with horseradish peroxidase (Sigma-Aldrich) and 3,3',5,5'-tetramethylbenzidine (Sigma-Aldrich) according to the manufacturer's recommendations. Between each step of the assay, wells were aspirated and rinsed thrice with PBS containing Tween-20 (Ambion Life Technologies). The optical density was measured at $650 \mathrm{~nm}$ using an ELISA plate reader (Synergy HT microplate reader, BioTek Instruments, Winooski, VT) and was proportional to the concentration of antibody against FimA, FimC, FimE, or SAP.

Case Definition. The concentrations of natural antibodies against fimbrial subunits determined by ELISA were correlated with the presence of other pathogens in cervical swab samples and occurrence of uterine diseases postpartum (Machado et al., 2014a,b). Briefly, metritis was defined based on the presence of fetid, watery, red-brown vaginal discharge retrieved after transrectal massage of the uterus associated with rectal temperature greater than $39.5^{\circ} \mathrm{C}$ at $6 \pm$ 1 DIM ( $\mathrm{n}=25 / 81)$. Clinical endometritis diagnosis was evaluated at $35 \pm 3$ DIM by visual inspection of uterine lavage. Uterine flush was performed using 20 $\mathrm{mL}$ of sterile saline solution and scored from 0 to 2 $(0=$ clear flush; $1=$ bloody flush without pus; $2=$ purulent or mucopurulent flush), with score 2 considered to be clinical endometritis $(n=7 / 80$; Machado et al., 2012b). Furthermore, uterine flush was cultured on Mueller-Hinton agar plates supplemented with $5 \%$ defibrinated sheep blood and presence of $T$. pyogenes were identified based on colony morphology, hemolysis, and Gram staining ( $\mathrm{n}=12 / 79)$. Cervical swabs collected at $2 \pm 1$ DIM were cultured on CHROMagar (Difco, Becton Dickinson) and the presence of E. coli was defined based on the observation of blue colonies $(\mathrm{n}=49 / 81)$. Additionally, the presence of the virulence factor from $E$. coli fim $H$ was detected by PCR as described previously ( $\mathrm{n}=8 / 76$; Machado et al., 2014a). Cervical swabs collected at $6 \pm 1$ DIM were cultured on LKV agar (Anaerobe Systems, Morgan Hill, CA) and presence of Fusobacterium necrophorum was evaluated based on colony morphology $(\mathrm{n}=39 / 78)$. The information in parentheses indicates the number of cows diagnosed as positive and the total number of cows evaluated for each condition.

\section{Statistical Analyses}

Concentrations of serum antibodies against fimbrial subunits were analyzed by ANOVA for repeated measures using the MIXED procedure of SAS version 9.3 (SAS Institute Inc., Cary, NC). Separate analyses were conducted for each condition (metritis, clinical endometritis, and the presence of E. coli, fim H, F. necrophorum, and $T$. pyogenes in the reproductive tract). The fixed effects of condition, day relative to calving, and the interaction between condition and day relative to calving were included in each statistical model and cow was considered a random effect. The heterogeneous first-order autoregressive covariance structure resulted in the smallest Schwarz's Bayesian information criterion value and was used in all analyses. Tests for normality of residuals and homogeneity of variances were conducted for each dependent variable. Results are presented as least squares means and standard error of the means. Differences with $P \leq 0.05$ were considered significant and those with $0.05<P \leq 0.10$ were considered tendencies. 


\section{RESULTS}

\section{Identification of Putative Operons Involved in Fimbrial Assembly}

Putative genes encoding for fimA, fim C, and fimE were located in ORF AHU90603, AHU90433, and AHU90532, respectively (Figure 1). Conversely, the fim $G$ gene was not identified in the genome of T. pyogenes TP6375. A base pair insert was identified in the sequencing data for ORF AHU90603, thus creating an early stop codon for the fimA annotated ORF. Because the remainder of the fimA gene is located in ORF AHU90604, its ORF will be referred to as AHU90603_4. In addition to the known fimbriae evaluated in the present study, a fourth putative major fimbria-encoding gene, namely fimJ, was identified in ORF AHU90586 through the evaluation of CWSS LPXTG motifs. The BLASTp was used to analyze the genes surrounding ORF AHU90603, AHU90433, AHU90532, and AHU90586 to predict putative functions. Genes with high AA similarity to proteins involved in fimbrial functions were considered to be part of a putative fimbrial operon. As a result, 4 putative fimbrial operons in $T$. pyogenes were identified, each containing 4 different genes related to a major fimbrial subunit, a putative upstream sortase, and 2 minor fimbrial subunits (Figure 1). Interestingly, all fimbria-related genes identified here for T. pyogenes TP6375 were also present in the genome of T. pyogenes MS249 (Goldstone et al., 2014).

\section{Sequence Alignment and Phylogenetic Analysis}

High similarity was observed between the 4 putative fimbrial proteins identified in the genome of T. pyogenes TP6375 and those described for T. pyogenes MS249 and other gram-positive species. Conserved motifs associated with known major fimbrial subunits of gram-positive bacteria were also identified. For each putative fimbrial operon, 3 regions required for proper major fimbriae protein function were identified, the pilin motif WxxxVxVYPKN with the invariant lysine (Figure 2A), the $\mathrm{E}$ box with the invariant glutamate (Figure 2B), and the CWSS in the $\mathrm{C}$ terminus comprising a LPXTG motif (Figure 2C). The pilin motif identified in FimJ and its top 4 hits showed an insertion varying from 5 to $8 \mathrm{AA}$ residues in the variable region between $\mathrm{W}$ and $\mathrm{V}$, located in the initial part of the motif. Accordingly, the role of FimJ as a major fimbrial subunit requires further evaluation. Neither an E box nor the pilin motif is present in minor fimbriae (Mandlik et al., 2007).

An evolutionary tree encompassing 30 sequences was constructed to depict relationships and differences among the putative fimbrial gene sequences (Figure
$3)$. The full alignment was 791 residues, whereas the masked alignment was 198 (25.3\%). In both alignments, the same higher-level groups were recovered; thus, only the full alignment phylogeny is presented. The BLASTp for FimC recovered FimA AHU90603 as one of the top matches and the BLASTp for FimA and FimC retrieved the same sequences. FimA and FimC translated sequences are sister to each other, and the top hits from each BLASTp search form a monophyletic group of mixed FimA and FimC matches. For FimE and FimJ, the top hits form 2 separate groups, and each fimbrial translated sequence is most closely related to putative proteins in other organisms. FimA and FimC exhibit less divergence than the FimE and FimJ pair.

\section{Antigenicity Against Fimbrial Protein in Dairy Cows}

Cows from which $T$. pyogenes was recovered by uterine flushing at $35 \pm 3$ DIM had consistently greater $(P$ $<0.01)$ concentrations of antibodies against FimC and FimE in serum compared with herdmates without $T$. pyogenes (Figure 4). The presence of T. pyogenes was not associated with changes in antibodies against FimA and SAP. However, the interaction between presence of $T$. pyogenes and day relative to calving was significant $(P \leq 0.01)$ and serum antibodies against FimA and SAP increased from $\mathrm{d}-30$ to 35 relative to calving in cows with T. pyogenes, whereas no change was observed in cows negative for $T$. pyogenes. The presence of E. coli, F. necrophorum, and fimH in cervical swabs postpartum and their interactions with day relative to calving did not affect $(P>0.10)$ serum concentrations of antibodies against FimA, FimC, FimE, and SAP. Serum antibodies against FimC and SAP tended to be affected $(P \leq 0.08)$ by the interaction between occurrence of metritis at $6 \pm 1$ DIM and day relative to calving (Figure 5A). A decline in serum antibodies against both proteins was observed between d 2 and 35 relative to calving in cows diagnosed with metritis, whereas a steady increase was observed in healthy counterparts. The concentrations of antibodies against FimC was consistently greater $(P=0.01)$ in cows diagnosed with clinical endometritis at $35 \pm 3$ DIM compared with healthy counterparts (Figure 5B). Serum antibodies against FimC and SAP were affected $(P \leq 0.08)$ by the interaction between the occurrence of clinical endometritis and day relative to calving, mostly because of an increase in the concentrations of antibodies against both proteins after parturition. The occurrence of metritis and clinical endometritis during early lactation, as well as their interactions with day relative to calving, did not affect $(P>0.25)$ serum concentrations of antibodies against FimA and FimE. 


\section{DISCUSSION}

Postpartum uterine diseases represent a substantial economic burden to the dairy industry worldwide because of costs associated with treatment, milk withdrawal, reduced productivity, impaired reproductive performance, and premature culling from the herd (Gilbert et al., 2005; LeBlanc, 2008). Although a critical evaluation of the effects of endometritis on cow comfort has not been conducted, metritis is a major concern regarding animal welfare (Stojkov et al., 2015). Recent studies indicate that vaccination against pathogens involved with metritis has the potential to reduce the incidence of uterine diseases (Machado et al., 2014b). The discovery of virulence factors closely related to the establishment of disease is expected to provide novel
(1)

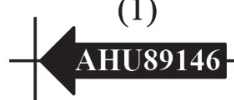

641,506

(5)

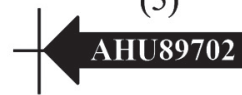

(6)

(2)

(3)

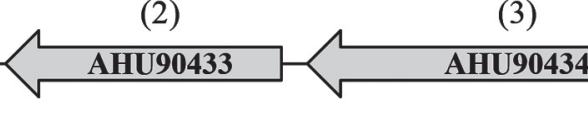

(7)

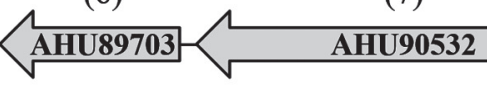

(4)

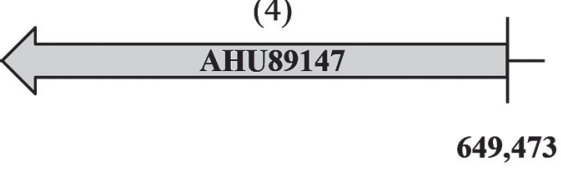

649,473

$1,393,066$

(8)

AHU90533

(9)

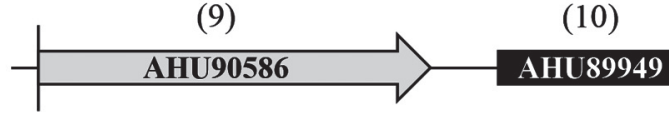

(11)

$1,750,700$

AHU90587

(12)

AHU90588

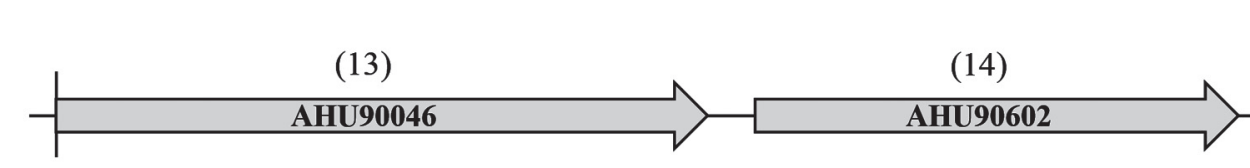

(14)

(15)

AHU90603 4 $>$ AHU90047-

$1,885,881$

$1,894,583$

\begin{tabular}{ccl}
\hline Seq. & Acession no. & Putative gene \\
\hline 1 & AHU89146 & FimC-sortase \\
2 & AHU90433 & FimC \\
3 & AHU90434 & Surface-anchored fimbrial subunit \\
4 & AHU89147 & Surface-anchored protein (SAP) \\
5 & AHU89702 & FimE-sortase \\
6 & AHU89703 & Fimbrial associated sortase-like protein \\
7 & AHU90532 & FimE \\
8 & AHU90533 & Surface-anchored fimbrial associated protein \\
9 & AHU90586 & FimJ \\
10 & AHU89949 & Fimbrial sortase \\
11 & AHU90587 & Surface-anchored protein \\
12 & AHU90588 & Surface-anchored fimbrial subunit \\
13 & AHU90046 & Collagen adhesin \\
14 & AHU90602 & Von Willebrand factor type A domain protein \\
15 & AHU90603_4 & FimA \\
16 & AHU90047 & FimA-sortase \\
\hline
\end{tabular}

Figure 1. Putative fimbria-related genes identified in the genome of Trueperella pyogenes strain TP6375. The numbers in bold below the drafts indicate the genomic location of starting and ending position of putative operons. Functions of putative genes were inferred by protein basic local alignment search tool (BLASTp). Each operon contains putative genes encoding 1 fimbriae-specific sortase (black arrow), and 1 major and 2 minor fimbriae subunits (gray arrows). 
targets for immunization and improve the efficacy of existing vaccines. Results from the present study depicted the presence of 4 putative major fimbrial subunits in the genome of $T$. pyogenes, including the newly described protein FimJ. Also, changes in serum concentrations of antibodies against selected fimbrial subunits, especially
A

$\uparrow$ FimA AHU90603_4 Trueperella pyogenes

AC WP_026420981 Actinobaculum urinale

AC WP_033512300 Bifidobacterium mongoliense

$\uparrow$ FimC AHU90433 Trueperella pyogenes

C KEI21998 Corynebacterium glutamicum ATCC14067

C WP_015650126 Corynebacterium callunae

$\uparrow$ FimE AHU90532 Trueperella pyogenes

E WP_016456076 Propionimicrobium lymphophilum

E WP_022864144 Varibaculum cambriense

$\uparrow$ FimJ AHU90586 Trueperella pyogenes

J WP_013169487 Arcanobacterium haemolyticum

J WP 016444500 Actinomyces europaeus

B

$\uparrow$ FimA AHU90603_4 Trueperella pyogenes

AC WP_026420981 Actinobaculum urinale

AC WP_033512300 Bifidobacterium mongoliense

$\dagger$ FimC AHU90433 Trueperella pyogenes

C KEI21998 Corynebacterium glutamicum ATCC14067

C WP_015650126 Corynebacterium callunae

$\dagger$ FimE AHU90532 Trueperella pyogenes

E WP_016456076 Propionimicrobium lymphophilum

E WP_022864144 Varibaculum cambriense

$\uparrow$ FimJ AHU90586 Trueperella pyogenes

J WP_013169487 Arcanobacterium haemolyticum

J WP_016444500 Actinomyces europaeus

$\mathrm{C}$

$\uparrow$ FimA AHU90603_4 Trueperella pyogenes

AC WP_026420981 Actinobaculum urinale

AC WP_033512300 Bifidobacterium mongoliense

$\uparrow$ FimC AHU90433 Trueperella pyogenes

C KEI21998 Corynebacterium glutamicum ATCC14067

C WP_015650126 Corynebacterium callunae

$\uparrow$ FimE AHU90532 Trueperella pyogenes

E WP_016456076 Propionimicrobium lymphophilum

E WP_022864144 Varibaculum cambriense

$\uparrow$ FimJ AHU90586 Trueperella pyogenes

J WP_013169487 Arcanobacterium haemolyticum

J WP_016444500 Actinomyces europaeus
${ }_{259}$ N EWV - - - - - - - - YDVHA YPKNKL T ${ }_{283}$ ${ }_{259} \mathrm{KNW}$ I - - - - - - - Y Y VHAY P KNQKG ${ }_{283}$ ${ }_{259} \mathrm{GNWL}-\ldots-\ldots$ ${ }^{259}$ N KWV - - - - - - - YDVHA Y P KNV L T ${ }_{283}$

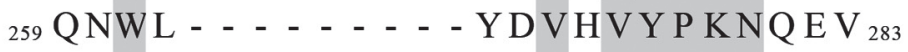

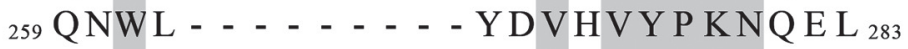
${ }^{259}$ D S WM - - - - - - - - YDVHVYPKNQAA ${ }_{283}$ ${ }_{259} \mathrm{~A} \mathrm{~S} \mathrm{WL} \mathrm{-} \mathrm{-} \mathrm{-} \mathrm{-} \mathrm{-} \mathrm{-} \mathrm{F} \mathrm{EADVYP} \mathrm{KNQVN} 283$ ${ }_{259} \mathrm{~K} A W N-\ldots-$ - - S DVHVY P KNQEN $_{283}$ ${ }_{259}$ N KW I T D L T T G - - KYA VWV Y P KN S L E ${ }_{283}$ ${ }_{259} \mathrm{~T}$ S W I MD - E E GNDKY A VYVY P KN S L D 283 ${ }_{259}$ S KW I MD - GK - - - NY G LWVYP KN S L E 283

${ }_{657}$ S D S KD YCLKE T AA P A G YV LD P V GR T 681 ${ }_{657}$ N P S K H Y C VY E T K V P A G Y S A S ADG I D 681 ${ }_{657}$ N P S KDYC LY E TKA P A G F I V G T F DHK 681 ${ }_{657}$ D A T KD YC LKE T A A P A G Y I LD S T G I D 681 ${ }_{657}$ N P NKDYC LY E TKA P S GYVA GP VQK - 681 ${ }_{657}$ DANKDYC LY E TKA P A GYVAGP S QV - 681 ${ }_{657}$ KQF Y S YCLV E TKS P D G YQLLAE P I E 681 ${ }_{657}$ - ADNTYCLVETKA P T GYQLLPKA T P 681 ${ }_{657}$ ANQVP Y C LVEEQA P A GYQ I L P QA I K 681 ${ }_{657}$ P QY S S YV LV E TKA P EKYELNKA P I P 681 ${ }_{657}$ - K L P EYC I VEKKA P AKYELRHDP I K 681 ${ }_{657}$ D T V P GYVLVETKA P A GYE L I P S P I D 681

${ }_{744}$ E N V KV E G - P D L P L T GA QG T A I MVV T 768 ${ }_{744}$ VN P KKDG - P DL P L T GAGGVLLL S I I ${ }_{768}$ ${ }_{744}$ T NVQQNH - P N L P L T GAAG T I VMT L G 768 ${ }_{744}$ D N V KV T G - P HL P L T GAQG T A L L TGA ${ }_{768}$ ${ }_{744}$ E N T K K D G - P N L P L T GGQG T LMMT I G 768 ${ }_{744}$ E N T KK E G - P N L P L T G GQG TMAMT VG ${ }_{768}$ ${ }_{744}$ V N Q P D N L KN K L P L T GG E G I A L V S V L ${ }_{768}$

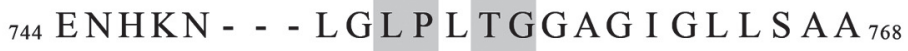
${ }_{744}$ T NHKN - - - L G L P L T GAQG I L LV S A L ${ }_{768}$ ${ }_{744}$ VN I P A K - - P KL PMT GGA GV A L F G I L 768 ${ }_{744}$ VN I P VK - - P K L PMT GGAGVA L F G F L 768 ${ }_{744}$ I NFEAL - - P KL P L T GGTGVALFG I L 768

Figure 2. Amino acid sequence alignment of putative fimbria-related genes identified in the genome of Trueperella pyogenes strain TP6375 (i.e., indicated by daggers) using the GenBank database, highlighting the pilin motif WxxxVxVYPKN with the invariant lysine (panel A), the $\mathrm{E}$ box with the invariant glutamate (panel B), and the CWSS in the C terminus comprising a LPXTG motif (panel C). Matches from T. pyogenes strain MS249 were excluded. 


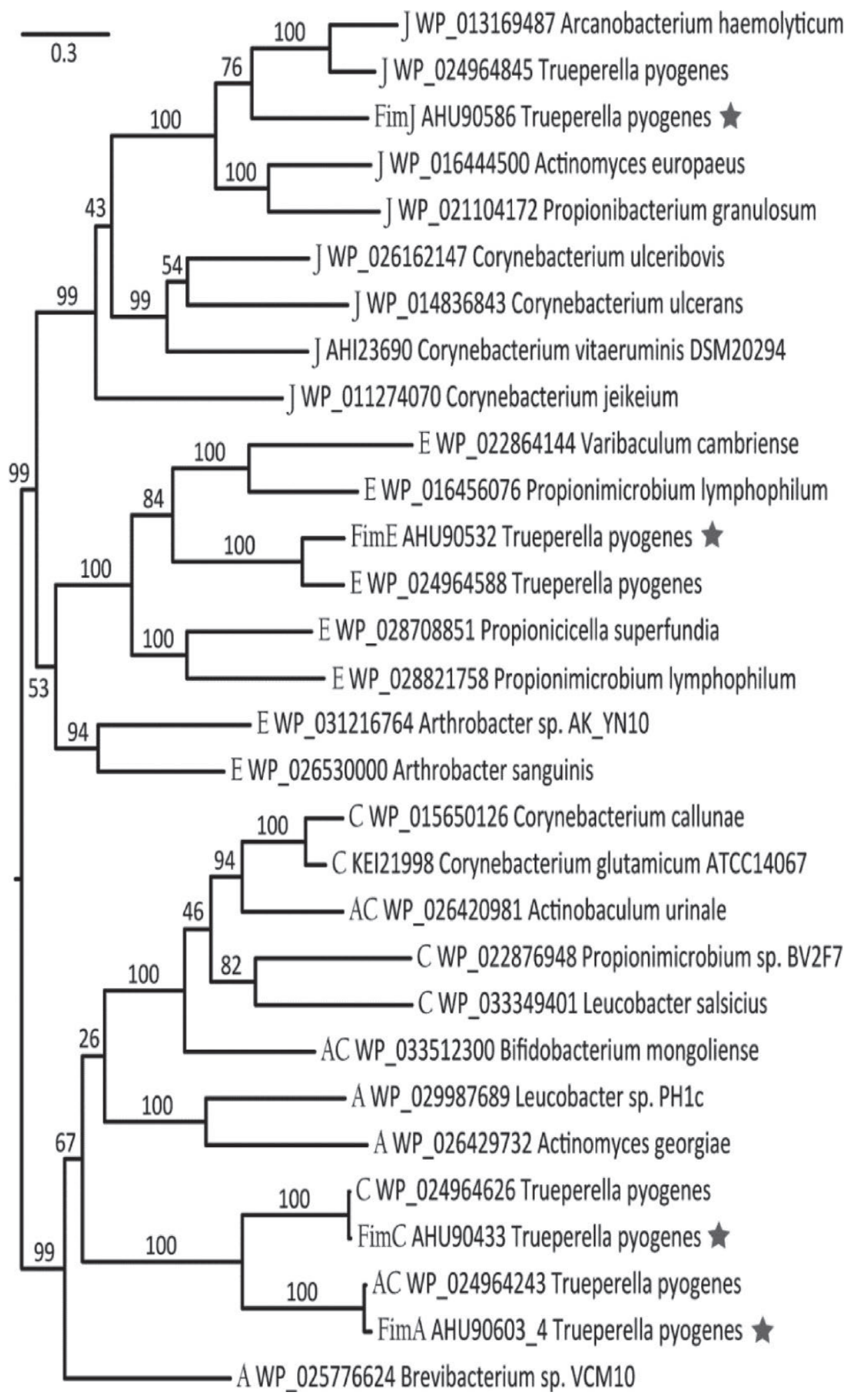

Figure 3. Phylogenetic tree of the evolutionary relationship among FimA, FimC, FimE, and FimJ. The scale bar defines the change represented per unit length. The letter before each GenBank accession number indicates the protein basic local alignment search tool (BLASTp) from which the referred sequence was retrieved. Stars indicate putative fimbria-related genes identified in the genome of Trueperella pyogenes strain TP6375 in the present study. 

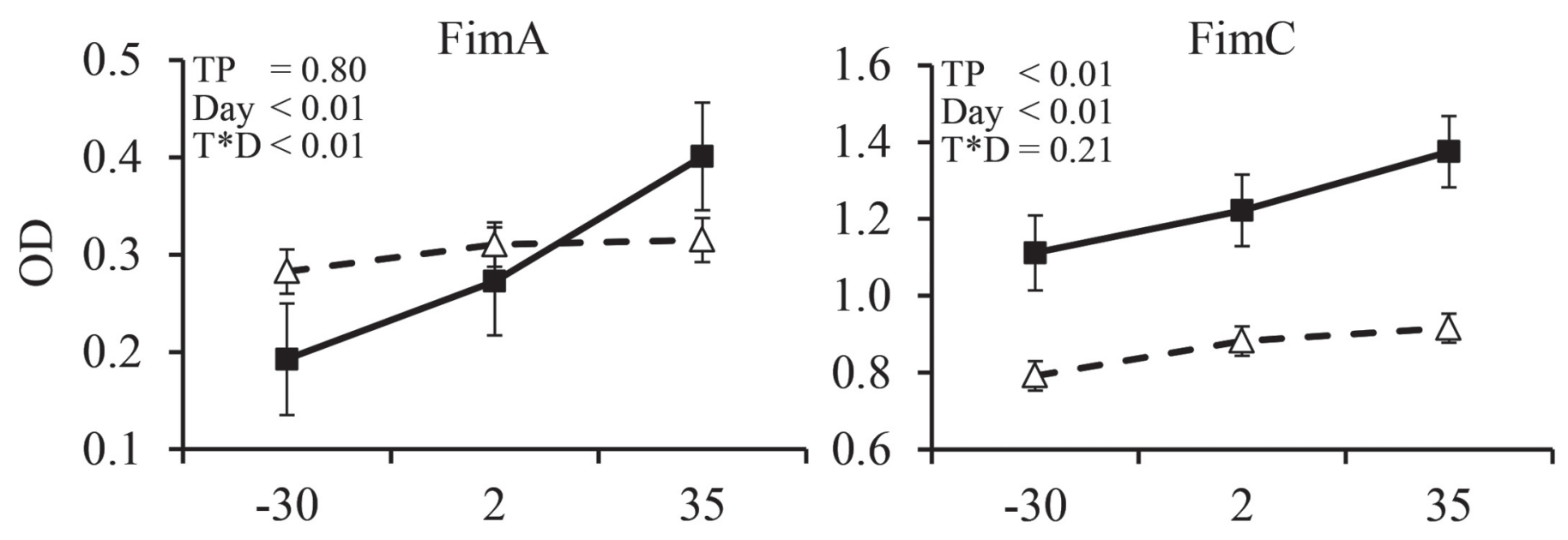

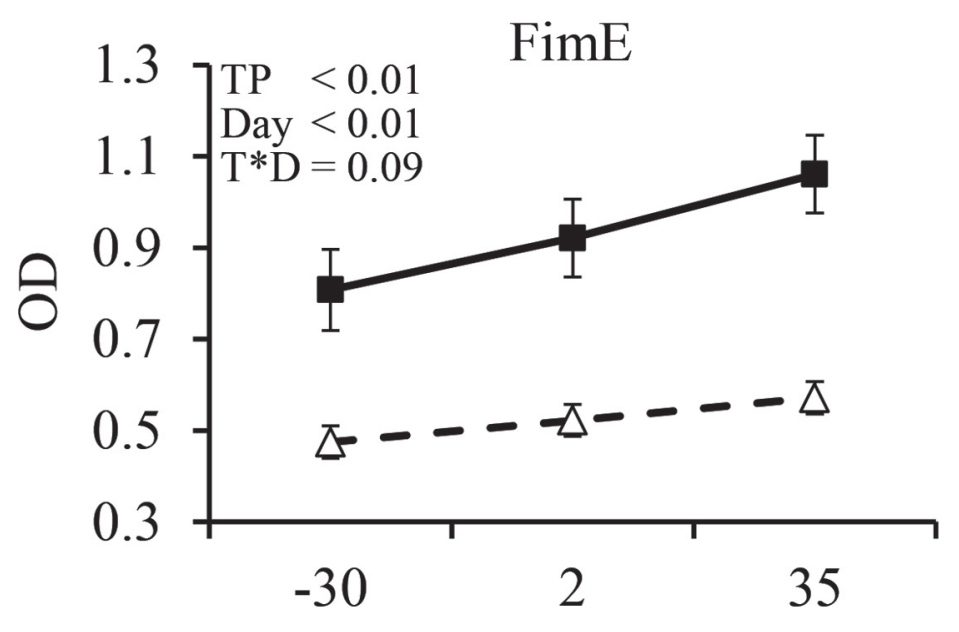

Day relative to calving

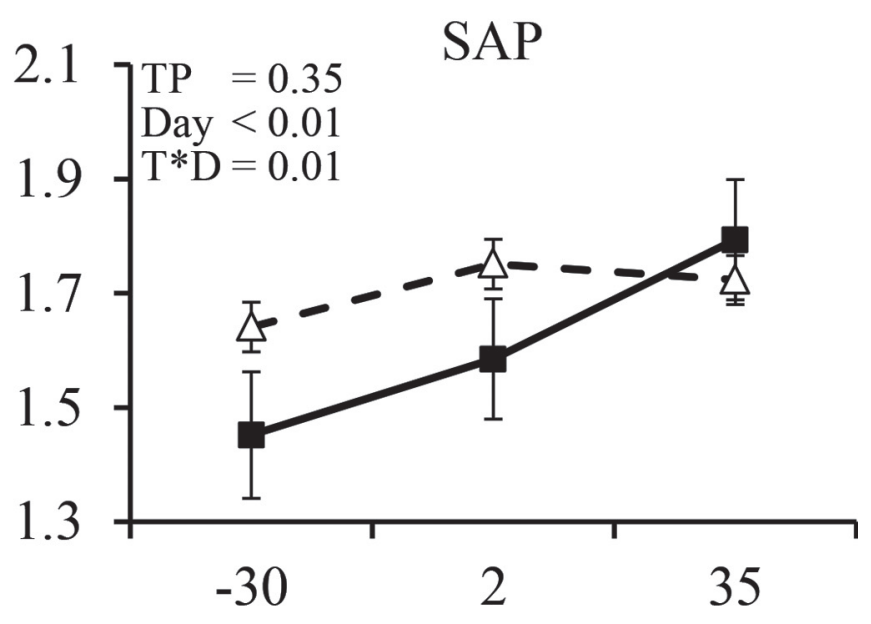

Day relative to calving

Figure 4. Serum concentrations of antibodies (measured by optical density, OD) against FimA, FimC, FimE, and surface-anchored protein (SAP) in cows with (solid squares, solid line) and without (open triangles, dashed line) Trueperella pyogenes present in uterine flushing collected on d $35 \pm 3$ postpartum. $\mathrm{TP}=$ presence of $T$. pyogenes, $\mathrm{T}^{*} \mathrm{D}=$ interaction between presence of $T$. pyogenes and day relative to calving.

FimC, suggest that T. pyogenes fimbriae are key antigens for mounting an immune response and potential targets for vaccine development.

Similar to other gram-positive bacteria (Scott and Zahner, 2006; Danne et al., 2011), fimbrial genes from T. pyogenes are gathered in clusters together with genes encoding for proteins required for pili assembly and functionality. For instance, a transpeptidase from the class $\mathrm{C}$ sortase family was identified in each fimbrial cluster in the present study. As opposed to type A or housekeeping sortases that are responsible for catalyzing the binding of fimbrial subunits to the bacterial cell wall, type $\mathrm{C}$ sortases catalyze fimbrial polymerization and the attachment of minor fimbrial proteins. This reaction involves the formation of an acyl enzyme inter- mediate between a conserved cysteine embedded in the sortase active site and the threonine of the LPxTG-like motif, which undergoes a nucleophylic attack by the lysine of the fimbrial motif and bridges together pilin subunits. For that reason, type $\mathrm{C}$ sortases are required for the polymerization of the pilus protein (Dramsi et al., 2006; Rosini et al., 2006). Similarly, the pilin motif WxxxVxVYPKN, the fimbrial motif WxxxVxVYPK, and the conserved E box motif YxLxETxAPxGY are sequences of AA necessary for proper polymerization of fimbrial subunits and were observed in each putative fimbrial operon. These findings further support the functionality of major fimbrial clusters identified in the genome of $T$. pyogenes. The 4 putative major fimbrial genes identified in the genome of $T$. pyogenes in the 
A
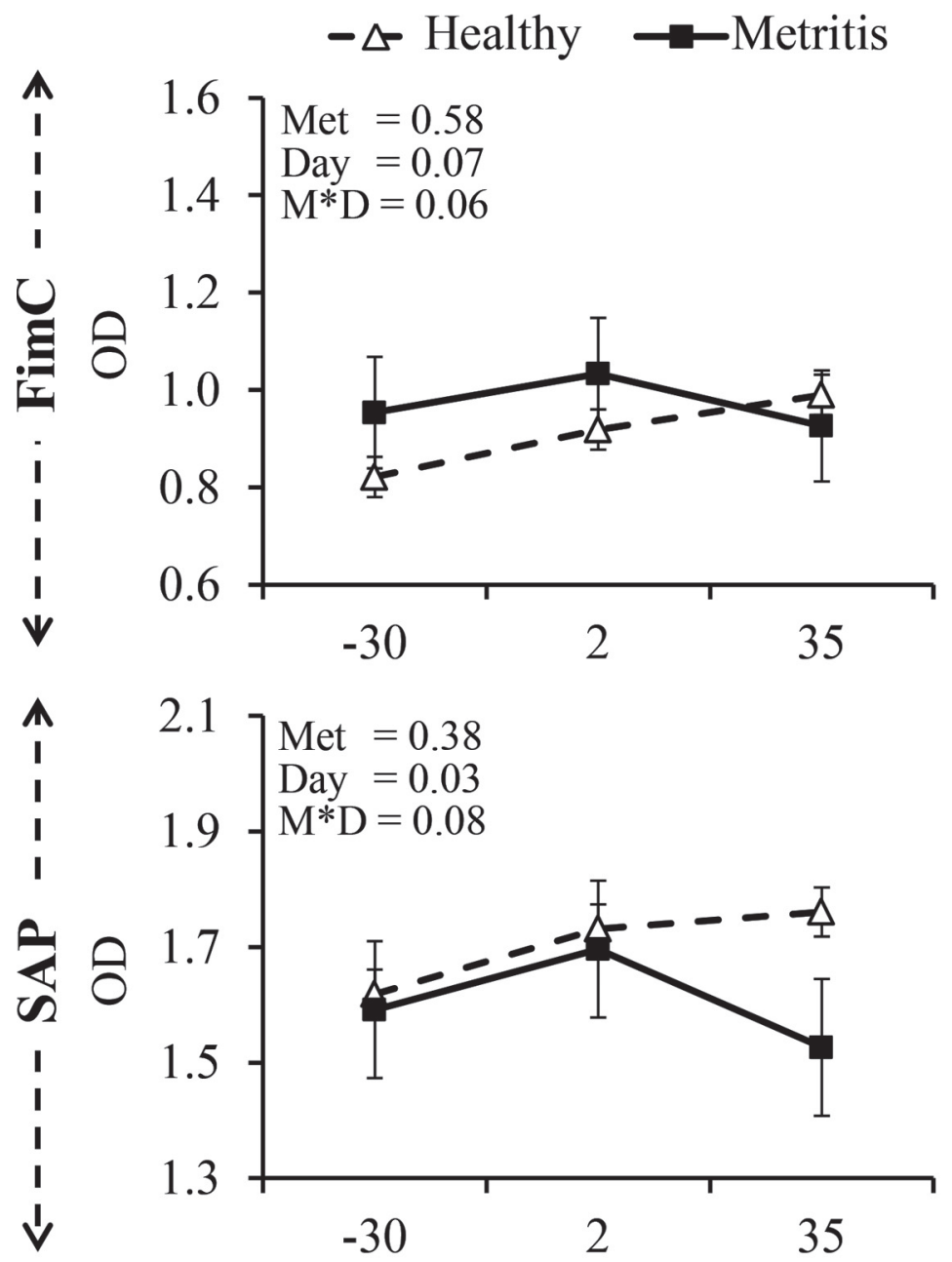

Day relative to calving
B

\section{$-\Delta$ Healthy $\rightarrow-$ Endometritis}
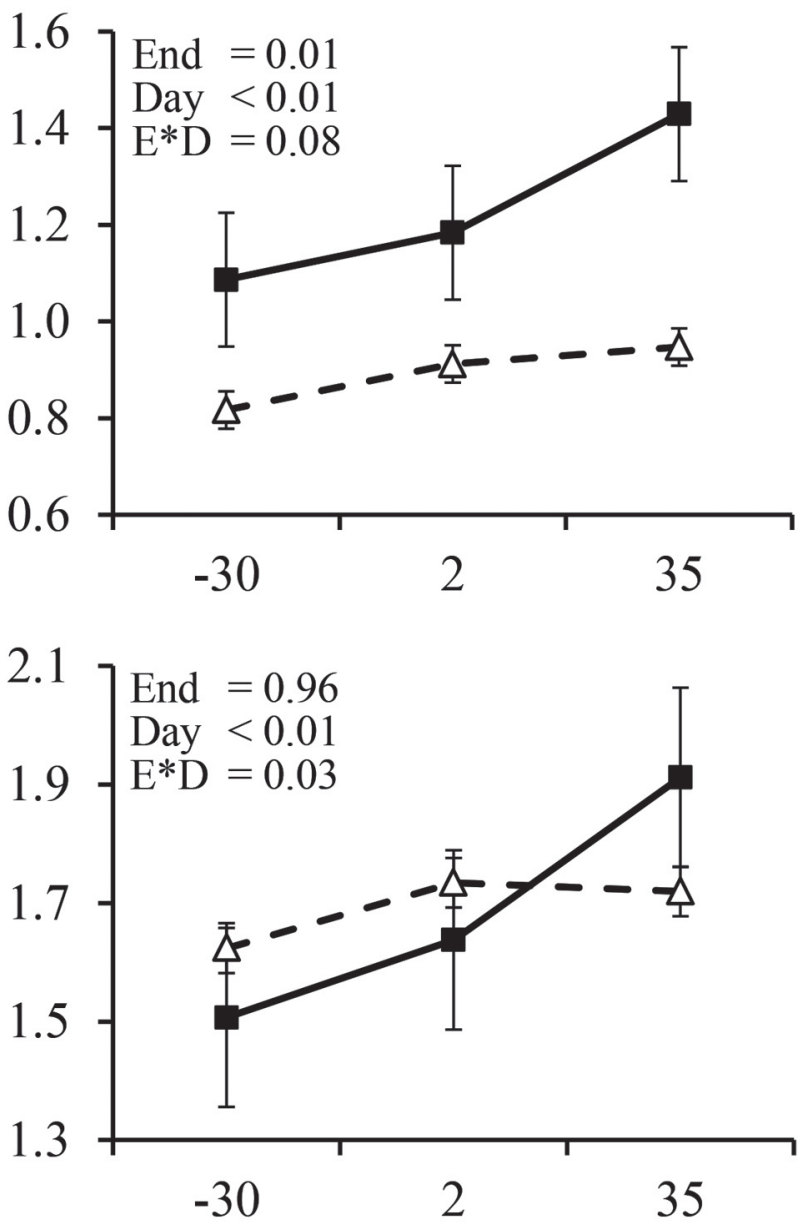

Day relative to calving

Figure 5. Relationship between serum concentrations of antibodies (measured by optical density, OD) against FimC and surface-anchored protein (SAP) and the occurrence of metritis (panel A) and clinical endometritis (panel B). Met $=$ occurrence of metritis evaluated at $6 \pm 1 \mathrm{~d}$ postpartum; $\mathrm{M}^{*} \mathrm{D}=$ interaction between occurrence of metritis and day relative to calving; End = occurrence of clinical endometritis evaluated at $35 \pm 3 \mathrm{~d}$ postpartum; $\mathrm{E}^{*} \mathrm{D}=$ interaction between occurrence of clinical endometritis and day relative to calving.

current study are supposedly related by duplication or lateral gene transfer. Based on the branch divergence and branch support values described here, these fimbrial proteins clustered into 2 distinct monophyletic groups. FimA and FimC were more closely related to each other and exhibited less pairwise divergence compared with FimE and FimJ, which encompassed a second phylogenetic group. It is interesting to note that the sequences for major fimbrial subunits aligned closely to those previously described for T. pyogenes.

Genes encoding collagen binding proteins and Von Willebrand domain A were found immediately upstream of the fimA gene. Collagen binding protein $\mathrm{A}$ has been previously described to interact with collagen types I, II, and IV to increase bacterial binding to both HeLa and 3T6 cells (Esmay et al., 2003). Moreover, Von Willebrand domain A-containing proteins are known to be involved in cell-to-cell adhesion and formation of multiprotein complexes. Neither of these proteins has been described as minor fimbrial subunits. However, each one contains a CWSS with hydrophobic N-terminal regions, positively charged tails, and an LPxTG-like motif that can be targeted by specific fimbrial class $\mathrm{C}$ sortases. The other putative minor fimbriae found in the present study have been described previously as surface-anchored fimbrial subunits. 
Increased concentrations of antibodies against FimC and FimE in cows with T. pyogenes present in the uterus at 35 DIM suggest that these fimbriae are involved in the persistency of the pathogen in the reproductive tract. As opposed to other virulence factor such as pyolysin and collagen-binding protein $\mathrm{A}$, genes encoding for the aforementioned fimbrial proteins were not observed in all isolates in previous studies (Silva et al., 2008). Of particular importance, FimC has been identified in only $67 \%$ of the isolates (Silva et al., 2008). Our results are in agreement with those from Silva et al. (2008) indicating that FimC and FimE are not associated with the occurrence of metritis. Nevertheless, the concentration of antibodies against FimC was greater in cows that developed clinical endometritis compared with healthy counterparts in the present study, underlining its possible role for maintenance of $T$. pyogenes in the uterus and development of uterine diseases later into lactation. Because SAP is the minor fimbria that putatively assembles together with FimC and both are encoded by the same operon, a similar pattern of expression would be expected for the 2 proteins. Serum concentrations of antibodies against SAP were not associated with uterine $T$. pyogenes postpartum in this study. However, circulating concentrations of antibodies against SAP increased from d 3 to 35 relative to calving in cows with $T$. pyogenes and in those diagnosed with clinical endometritis, suggesting that the expression of $\mathrm{SAP}$ is important for $T$. pyogenes persistency in utero and the pathogenesis of clinical endometritis. Because the study outlined here was not designed to evaluate fertility outcomes, further investigation is required to define the importance of FimC and SAP for long-term reproductive success and determine the efficacy of immunization against these proteins for prevention of endometritis in dairy cows.

\section{CONCLUSIONS}

Genes encoding for fimbrial proteins are present in the genome of $T$. pyogenes. Four putative fimbrial assembly clusters were identified, including the novel major fimbrial subunit FimJ. This newly discovered fimbrial protein presents greater similarity to FimE compared with FimA and FimC. The evaluation of serum antibodies against selected fimbrial subunits supports their importance for maintenance of uterine infection with $T$. pyogenes and for the establishment of uterine diseases in dairy cows. Nevertheless, additional research is necessary to evaluate the effect of antibodies against fimbrial subunits on fertility outcomes, as well as to determine the effectiveness of selected recombinant fimbrial proteins as a target for development of vaccines against uterine diseases in dairy cattle.

\section{ACKNOWLEDGMENTS}

The authors acknowledge funding from $\mathrm{CNPq}$ (National Council for Scientific and Technological Development, Brazil) to support José Carlos Oliveira Filho.

\section{REFERENCES}

Amos, M. R., G. D. Healey, R. J. Goldstone, S. M. Mahan, A. Düvel, H. J. Schuberth, O. Sandra, P. Zieger, I. Dieuzy-Labaye, D. G. Smith, and I. M. Sheldon. 2014. Differential endometrial cell sensitivity to a cholesterol-dependent cytolysin links Trueperella pyogenes to uterine disease in cattle. Biol. Reprod. 90:54.

Bicalho, M. L., V. S. Machado, G. Oikonomou, R. O. Gilbert, and R. C. Bicalho. 2012. Association between virulence factors of Escherichia coli, Fusobacterium necrophorum, and Arcanobacterium pyogenes and uterine diseases of dairy cows. Vet. Microbiol. 157:125-131.

Danne, C., J. M. Entenza, A. Mallet, R. Briandet, M. Debarbouille, F. Nato, P. Glaser, G. Jouvion, P. Moreillon, P. Trieu-Cuot, and S. Dramsi. 2011. Molecular characterization of a Streptococcus gallolyticus genomic island encoding a pilus involved in endocarditis. J. Infect. Dis. 204:1960-1970.

Doré, E., G. Fecteau, P. Helie, and D. Francoz. 2007. Liver abscesses in Holstein dairy cattle: 18 cases (1992-2003). J. Vet. Intern. Med. 21:853-856.

Dramsi, S., E. Caliot, I. Bonne, S. Guadagnini, M. C. Prevost, M. Kojadinovic, L. Lalioui, C. Poyart, and P. Trieu-Cuot. 2006. Assembly and role of pili in group B streptococci. Mol. Microbiol. 60:1401-1413.

Esmay, P. A., S. J. Billington, M. A. Link, J. G. Songer, and B. H. Jost. 2003. The Arcanobacterium pyogenes collagen-binding protein, $\mathrm{CbpA}$, promotes adhesion to host cells. Infect. Immun. 71:4368-4374.

Gilbert, R. O., S. T. Shin, C. L. Guard, H. N. Erb, and M. Frajblat. 2005. Incidence of endometritis and its effects on reproductive performance of dairy cows. Theriogenology 64:1879-1888.

Goldstone, R. J., M. Amos, R. Talbot, H. J. Schuberth, O. Sandra, I. M. Sheldon, and D. G. Smith. 2014. Draft genome sequence of Trueperella pyogenes, isolated from the infected uterus of a postpartum cow with metritis. Genome Announc. 2. http://dx.doi. org/10.1128/genomeA.00194-14.

Jost, B. H., and S. J. Billington. 2005. Arcanobacterium pyogenes: Molecular pathogenesis of an animal opportunist. Antonie van Leeuwenhoek 88:87-102.

Jost, B. H., J. G. Songer, and S. J. Billington. 1999. An Arcanobacterium (Actinomyces) pyogenes mutant deficient in production of the pore-forming cytolysin pyolysin has reduced virulence. Infect. Immun. 67:1723-1728.

Katoh, K., and D. M. Standley. 2013. MAFFT multiple sequence alignment software version 7: Improvements in performance and usability. Mol. Biol. Evol. 30:772-780.

Kück, P., K. Meusemann, J. Dambach, B. Thormann, B. M. von Reumont, J. W. Wagele, and B. Misof. 2010. Parametric and nonparametric masking of randomness in sequence alignments can be improved and leads to better resolved trees. Front. Zool. 7:10.

LeBlanc, S. J. 2008. Postpartum uterine disease and dairy herd reproductive performance: A review. Vet. J. 176:102-114.

Machado, V. S., M. L. Bicalho, R. O. Gilbert, and R. C. Bicalho. 2014a. Short communication: Relationship between natural antibodies and postpartum uterine health in dairy cows. J. Dairy Sci. 97:7674-7678.

Machado, V. S., M. L. Bicalho, E. B. Meira Junior, R. Rossi, B. L. Ribeiro, S. Lima, T. Santos, A. Kussler, C. Foditsch, E. K. Ganda, G. Oikonomou, S. H. Cheong, R. O. Gilbert, and R. C. Bicalho. 2014b. Subcutaneous immunization with inactivated bacterial components and purified protein of Escherichia coli, Fusobacterium necrophorum, and Trueperella pyogenes prevents puerperal metritis in Holstein dairy cows. PLoS ONE 9:e91734. 
Machado, V. S., M. L. Bicalho, R. V. Pereira, L. S. Caixeta, J. H. Bittar, G. Oikonomou, R. O. Gilbert, and R. C. Bicalho. 2012a. The effect of intrauterine administration of mannose or bacteriophage on uterine health and fertility of dairy cows with special focus on Escherichia coli and Arcanobacterium pyogenes. J. Dairy Sci. 95:3100-3109.

Machado, V. S., and R. C. Bicalho. 2014. Complete genome sequence of Trueperella pyogenes, an important opportunistic pathogen of livestock. Genome Announc. 2. http://dx.doi.org/10.1128/ genomeA.00400-14.

Machado, V. S., W. A. Knauer, M. L. Bicalho, G. Oikonomou, R. O. Gilbert, and R. C. Bicalho. 2012b. A novel diagnostic technique to determine uterine health of Holstein cows at 35 days postpartum. J. Dairy Sci. 95:1349-1357.

Maione, D., I. Margarit, C. D. Rinaudo, V. Masignani, M. Mora, M. Scarselli, H. Tettelin, C. Brettoni, E. T. Iacobini, R. Rosini, N. D'Agostino, L. Miorin, S. Buccato, M. Mariani, G. Galli, R. Nogarotto, V. Nardi-Dei, F. Vegni, C. Fraser, G. Mancuso, G. Teti, L. C. Madoff, L. C. Paoletti, R. Rappuoli, D. L. Kasper, J. L. Telford, and G. Grandi. 2005. Identification of a universal Group B streptococcus vaccine by multiple genome screen. Science 309:148-150.

Mandlik, A., A. Swierczynski, A. Das, and H. Ton-That. 2007. Corynebacterium diphtheriae employs specific minor pilins to target human pharyngeal epithelial cells. Mol. Microbiol. 64:111-124.

Mandlik, A., A. Swierczynski, A. Das, and H. Ton-That. 2008. Pili in Gram-positive bacteria: Assembly, involvement in colonization and biofilm development. Trends Microbiol. 16:33-40.

Miller, M. A., T. Schwartz, B. E. Pickett, S. He, E. B. Klem, R. H. Scheuermann, M. Passarotti, S. Kaufman, and M. A. O'Leary. 2015. A RESTful API for access to phylogenetic tools via the CIPRES Science Gateway. Evol. Bioinform. Online 11:43-48.

Nuccitelli, A., C. D. Rinaudo, B. Brogioni, R. Cozzi, M. Ferrer-Navarro, D. Yero, J. L. Telford, G. Grandi, X. Daura, M. Zacharias, and D. Maione. 2013. Understanding the molecular determinants driving the immunological specificity of the protective pilus $2 \mathrm{a}$ backbone protein of group B Streptococcus. PLOS Comput. Biol. 9:e1003115.

Petersen, T. N., S. Brunak, G. von Heijne, and H. Nielsen. 2011. SignalP 4.0: Discriminating signal peptides from transmembrane regions. Nat. Methods 8:785-786.
Ribeiro, M. G., R. M. Risseti, C. A. Bolanos, K. A. Caffaro, A. C. de Morais, G. H. Lara, T. O. Zamprogna, A. C. Paes, F. J. Listoni, and M. M. Franco. 2015. Trueperella pyogenes multispecies infections in domestic animals: A retrospective study of 144 cases (2002 to 2012). Vet. Q. 35:82-87.

Rosini, R., C. D. Rinaudo, M. Soriani, P. Lauer, M. Mora, D. Maione, A. Taddei, I. Santi, C. Ghezzo, C. Brettoni, S. Buccato, I. Margarit, G. Grandi, and J. L. Telford. 2006. Identification of novel genomic islands coding for antigenic pilus-like structures in Streptococcus agalactiae. Mol. Microbiol. 61:126-141.

Santos, T. M., L. S. Caixeta, V. S. Machado, A. K. Rauf, R. O. Gilbert, and R. C. Bicalho. 2010. Antimicrobial resistance and presence of virulence factor genes in Arcanobacterium pyogenes isolated from the uterus of postpartum dairy cows. Vet. Microbiol. 145:84-89.

Scott, J. R., and D. Zahner. 2006. Pili with strong attachments: Grampositive bacteria do it differently. Mol. Microbiol. 62:320-330.

Sens, A., and W. Heuwieser. 2013. Presence of Escherichia coli, Trueperella pyogenes, alpha-hemolytic streptococci, and coagulase-negative staphylococci and prevalence of subclinical endometritis. J. Dairy Sci. 96:6347-6354.

Silva, E., M. Gaivão, S. Leitão, B. H. Jost, C. Carneiro, C. L. Vilela, L. Lopes da Costa, and L. Mateus. 2008. Genomic characterization of Arcanobacterium pyogenes isolates recovered from the uterus of dairy cows with normal puerperium or clinical metritis. Vet. Microbiol. 132:111-118.

Stamatakis, A. 2014. RAxML version 8: A tool for phylogenetic analysis and post-analysis of large phylogenies. Bioinformatics 30:13121313.

Stojkov, J., M. A. von Keyserlingk, J. N. Marchant-Forde, and D. M. Weary. 2015. Assessment of visceral pain associated with metritis in dairy cows. J. Dairy Sci. 98:5352-5361.

Talavera, G., and J. Castresana. 2007. Improvement of phylogenies after removing divergent and ambiguously aligned blocks from protein sequence alignments. Syst. Biol. 56:564-577.

Williams, E. J., D. P. Fischer, D. U. Pfeiffer, G. C. England, D. E. Noakes, H. Dobson, and I. M. Sheldon. 2005. Clinical evaluation of postpartum vaginal mucus reflects uterine bacterial infection and the immune response in cattle. Theriogenology 63:102-117. 